\title{
Nature of friction in extrusion process and its effect on material flow
}

\author{
I. Flitta and T. Sheppard
}

This investigation focuses on simulation of the extrusion process and in particular the effect of the initial billet temperature on friction and its consequences on material flow. The simulation is compared with data obtained from an experimental extrusion press. All the simulations are performed with the implicit finite element codes FORGE2 and FORGE3. The effect of the initial billet temperature on the deformation zone pattern and its consequent effect on friction using both numerical simulation and experimental work are presented. A comparison with experiments is made to assess the relative importance of some extrusion parameters in the extrusion process and to ensure that the numerical discretisation provided a true simulation of the process. A specific functional relationship to directly measure interfacial friction under conditions approaching those encountered in the quasi-static deformation process is described. The results revealed that the friction factor increases with increase in initial billet temperature and varies from 0.65 at $300 \mathrm{C}$ to a 0.91 at $450 \mathrm{C}$ after reaching the peak pressure. The dead metal zone is observed to vary in form and has a greater volume at high temperatures. The increase in friction results in an increase of initial extrusion load. The finite element program appears to predict all the major characteristics of the flow observed macroscopically.

MST/5520

The authors are in Bournemouth University, 12 Christchurch Road, Studland House Bournemouth, Dorset BH1 3 NA, UK (Iflita@bournemouth.ac.uk; tsheppard@bournemouth.ac.uk). Manuscript received 15 May 2002; accepted 16 December 2002. (C) 2003 Io M Communications Ltd. Published by Maney for the Institute of Materials, Minerals and Mining.

\section{Introduction}

The basic process of extrusion is well described as a thermomechanical event in a quite recent text, ${ }^{1}$ which indicates that the mathematical description of the process is still largely semiempirical. The extrusion process is complex, involving interaction between the process variables and the material's high temperature properties. Theoretically, the process variables that can be controlled are the extrusion ratio $R$, the ram speed $V$, and the initial extrusion temperature $T$. However, events on the micromechanical scale are still not adequately described. The most important of these is possibly the mechanics at the interface between tooling and material. This influences the analyses of the temperature changes occurring during the process, the final temperature and the temperature history determining the structure of the extrudate and hence, to a large extent, its properties.

Most authors agree that one of the most significant parameters to define when modelling extrusion processes is the friction coefficient. ${ }^{1-6}$ The mechanism of interfacial friction in aluminium extrusion is very complex and is not a well understood phenomenon. Owing to the high pressures and temperatures that are necessary features of the process, experimentation is difficult and this combined with only a rough estimation of the relevant parameters, indicates that an accurate simulation might be of some use.

In the industrial context, assumptions are made that sticking friction prevails at the interface between the billet and the tools, and its value is a constant for all extrusion temperatures. Various studies and analytical methods have been used to establish the complex relationship between the extrusion parameters, friction, temperature, stress, strain, strain rate, etc. Some of these studies ${ }^{7-11}$ provided some understanding into the mechanisms that govern friction but fall short in supplying sufficient information to identify the mechanisms that can be applied quantitatively to model friction for the extrusion process.

Other workers ${ }^{1,9,10,12-17}$ have expressed the friction coefficient to be a constant and to range between 0.8 and 1 based on experimental observations, using either varying billet lengths or by measurement of the slope of the pressure-displacement locus. Variations of pressure with billet lengths have been investigated for 1100 and 2014 aluminium alloys, ${ }^{1,18}$ by extruding billets of varying lengths under identical extrusion conditions. It was observed that the peak pressure-billet length relationship in each case was linear, and the friction coefficient value determined from the data was $0 \cdot 88$ for AA 1100 and $0 \cdot 8$ for AA 2014 . The work indicated that the friction coefficient has an approximately constant value during the extrusion process regardless of the initial extrusion temperature.

Clearly neither of these methods of establishing the value of friction is satisfactory and for design purposes, the friction is usually assumed to be 0.85 . However, such measurements fail to take into account the differences in temperature at differing locations during the ram stroke and the consequent deviation in the flow stress. The extrusion pressure is significantly influenced by the temperature gradients modified in the billet during transfer to the container, and after upsetting in the container. Metal flow in the extrusion process is an important factor controlling the mechanical and structural properties of the extruded product.

The modelling of aluminium extrusion must be based on a fundamental understanding of the thermomechanical phenomena occurring during the process. While the problem is thus one of broader complexity, the derivation of systematic data from which it can be studied is rendered very difficult under industrial conditions. It is also difficult to quantify by experimental techniques. Accurate thermal and microstructural data of materials and in particular the interface data such as friction and heat transfer properties must be available. The accuracy of the results are not only sensitive to the geometric definition of the tooling and the input of the material rheological data under investigation, but they are also highly sensitive to the data input for the boundary conditions between the billet and the tooling. These parameters are clearly extremely sensitive when attempting to simulate the extrusion process. In early software development, requirements were only confined to a graphical illustration of the change in geometrical shape of the workpiece and an approximate value of pressure to 
undertake the process. In such cases, the accuracy of the data input, i.e. friction, heat transfer etc., were not necessarily high and consisted of an approximation to the yield stress and a global, averaged friction factor. With the ever increasing use of finite element method (FEM) techniques in the last few years to the extrusion process, the necessity for accurate input data becomes increasingly crucial. The analytical ability of current finite element codes enable the continuum parameters of the extrusion process, stress and strain histories and metal flow to be predicted in detail as deformation continues. In order to enable full advantage to be taken of the use of finite element programs available to simulate manufacturingprocesses to be exploited, not only are detailed mechanical, thermal and microstructural constitutive descriptions of materials required but mechanical and thermal properties of workpiece/tool interfaces and the tools also must be available. However, the definition of the interfacial friction in most of the current programs is one of the boundary conditions required for a solution to the problem. To overcome this problem a coupled experiment with inverted analytical solutions would inevitably obtain reliable data describing the friction phenomena of the process.

\section{Friction}

The coefficient of friction at the metal/billet interface contributes significantly to the complexity of extruding, and it is a point where the friction resistance approaches the shear resistance of the hot material during deformation. Furthermore, it is a point where a fraction or all of the displacement of the billet at the interface occurs by shear in its surface layers leaving a fragment of the billet deposited on the wall of the container. In practice, aluminium alloys are extruded without any lubricant or with only, a small amount of graphite applied to the die face. Finding a suitable lubricant would be a difficult task and in any case unlubricated aluminium extrusion is desirable in order to prevent impurity pick up from the tools and to ensure that all the material making up the extrudate surfaces originates from virgin material within the billet. Hence, the interfacial conditions at the billet/container interface during extrusion has a direct effect on metal flow, the stresses acting upon both the tools and within the material, and hence load and energy requirements and extrudate temperature.

During the extrusion process, the force necessary to overcome the friction between the billet and the container results in an increase in overall pressure to extrude. At the extreme condition between the billet and the container, friction at the interface cannot exceed the shear strength of the material. This extreme condition is termed sticking friction and can be represented generally as

$$
\tau_{\mathrm{F}}=\tau_{\max }
$$

where $\tau_{\mathrm{F}}$ is the interfacial friction and $\tau_{\mathrm{m} \text { ax }}$ is the shear yield stress of the billet material. The shear stress can be defined either by the Tresca yield criterion

$$
\tau_{\max }=\bar{\sigma} / 2
$$

where $\bar{\sigma}$ is the mean equivalent yield stress, or by a Von Mises material when the shear strength can be expressed as

$$
\tau_{\max }=\bar{\sigma} / \sqrt{3}
$$

where $\bar{\sigma} / \sqrt{3}$ is the mean equivalent shear flow stress. A modification to sticking friction is often introduced to account for the fact that friction forces are seldom as high as the shear strength of a material. The friction can be defined using what is sometimes referred to as the Tresca friction law, which assumes proportionality between the friction and the current shear flow stress of the material and can be written

$$
\tau_{\mathrm{F}}=\bar{m} \tau_{\max }
$$

$\bar{m}$ is the factor of proportionality and is commonly referred to as a friction factor and varies between $\bar{m}=0$ for perfect lubrication and $\bar{m}=1$ for sticking friction. The Tresca law treats the interface friction as pressure independent and relates the friction stress directly to the shear flow stress of the deformed material. The value of $\tau_{\max }$ can be determined from either Tresca or Von Mises yield functions, or from other descriptions of the plastic flow stress in shear.

The FEM used in metal forming can be generally categorised into viscoplastic plastic FEM and elastic viscoplastic plastic FEM, depending on which material constitutive equations are used. For extrusion of hot aluminium alloys, viscoplastic FEM is adopted to simulate the process because plastic strain dominates the process. For the convenience of simulation and modelling purposes, friction is considered in terms of the relative velocity between two surfaces, i.e. billet and the tooling. The friction law may be considered by assuming the Tresca friction law criteria described in equation (4) and is written as follows

$$
\tau=-m \bar{\sigma}_{\mathrm{s}} / \sqrt{3}
$$

At the limit when $\bar{m}$ is of unit value, all deformation will be in the form of shear in the subcutaneous regions of the billet rather than sliding against the tools. However, this simple representation is not able to include the contribution of local conditions such as temperature, pressure, surface quality and geometry. Otherwise, with the description and the inclusion of heat transfer, the value of $\bar{m}$ is defined with the same value as a constant, and it would be more realistic to specify this constant as a function of process variables. Although $\bar{m}$ should be assumed as a constant value, this formulation can be more useful and more sophisticated by allowing the friction coefficient or the friction factor to be functions of several variables.

\section{Finite element models and source of experimental data}

\section{GOVERNING EQUATIONS}

In this investigation, a commercial finite element package FORGE2 and FORGE3* (Refs. 19 and 20) developed by Transvalor (France) was used to simulate the extrusion of aluminium alloys. The data structure of the program includes the governing equations, the finite element of the workpiece, the rheology of the material, the tooling description, the frictional interface, and the numerical parameters to be defined in the iterative solution. The program uses implicit FEM to calculate the hot working parameters: load, strain rate, temperature rise, and deformation.

The temperature evolution is governed by internal heat conduction and internal heat dissipation under the constraints defined on the area boundary in terms of interchange (radiation, conduction and convection) and in terms of imposed temperature. This evolution is represented by the following heat equation

$$
\rho c \frac{\partial T}{\partial t}=\operatorname{div}(k \operatorname{grad} T)+Q
$$

where $\rho$ is the density, $c$ the specific heat, $T$ is the temperature, $Q$ is the internal heat dissipation generated by plastic deformation and $k$ is the conductivity. The temperature field in the billet or in the die is computed using equation (6). The work generated by the plastic deformation is largely dissipated in terms of heat inside the body leading to some energy being retained within the material.

*FORGE2 and FORGE3 are registered trademarks. 
In addition to the above governing equation, boundary conditions to describe geometry and friction are required. Friction can be dealt with by using equation (5).

In the FORGE2 and FORGE3 programs, the NortonHoff law describes the default rheology of the material. However, in order to obtain reliable results with a high degree of confidence, it is important to reduce the errors owing to approximate data used to describe the material behaviour. Experience shows that some differences can appear between the numerical predictions and experimental results. Part of these variations result from discretisation of the problem (FEM), however, inaccuracy often occurs from using data not calculated for the specific alloy over the complete working range. The most widely used equation to describe the deformation of aluminium alloys, is that proposed by Sellars and Tegart ${ }^{21}$ and subsequently modified by Sheppard and Wright ${ }^{22}$ to yield the steady state flow stress from the equation

$$
Z=A[\sinh (\alpha \bar{\sigma})]^{\mathrm{n}}=\dot{\bar{\varepsilon}} \exp \left(\frac{\Delta H}{G T}\right)
$$

from which

$$
\bar{\sigma}=\alpha^{-1} \ln \left\{(Z / A)^{1 / \mathrm{n}}+\left[(Z / A)^{2 / \mathrm{n}}+1\right]^{1 / 2}\right\}
$$

where $Z$ is termed the temperature compensated strain rate $\left(\mathrm{s}^{-1}\right) ; \Delta H$ is the activation energy for deformation $\left(\mathrm{kJ} \mathrm{mol}^{-1}\right)$; $G$ is the universal gas constant $\left(8.314 \mathrm{~J} \mathrm{~mol}^{-1} \mathrm{~K}^{-1}\right) ; \dot{\bar{\varepsilon}}$ is the mean equivalent strain rate $\left(\mathrm{s}^{-1}\right) ; A\left(\mathrm{~s}^{-1}\right)$ and $n$ are constants; $\alpha$ is a constant $\left(\mathrm{m}^{2} \mathrm{MN}^{-1}\right)$; and $T$ is the initial billet temperature $(\mathrm{K})$.

\section{PROCESS CONDITIONS}

The chemical compositions of the alloys used in this investigation are given in Table 1 . All the experimental data are extracted from the literature. ${ }^{16,17}$ Experiments were performed on a $5 \mathrm{MN}$ press vertically mounted with a heated container. The main ram was driven by a hydraulic pump during the extrusion cycle. The load was measured by Mayes load cell situated directly above the ram, the output from the cell being recorded on a Labmaster data recorder. Ram displacement and speeds were measured by a rectilinear potentiometer fixed between the moving crossheads and the press bolster.

Constant ram speeds of $v=3$ and $8 \mathrm{~mm} \mathrm{~s}^{-1}$ were imposed with an extrusion ratio of 30:1 for AA 2024 and $\mathrm{Al}-1 \mathrm{wt}-\% \mathrm{Cu}$ respectively. In FORGE2 program, only half of the cross-section requires modelling due to symmetry. A flat faced die with a $5 \mathrm{~mm}$ die bearing length was used for all simulations. The boundary conditions at the billet/container interface were evaluated by the Tresca friction type mechanism. The coefficient of friction was varied from 0.5 to 0.99 in order to simulate conditions ranging from low stick-slip to fully sticking conditions. The container and die temperatures were $50 \mathrm{~K}$ below the initial billet temperature for all the analyses in an attempt to reduce the temperature of the extrudate and hence, to simulate industrial conditions.

The data describing the material behaviour available to the authors were obtained using Torque Twist data from torsion tests and optimised to obtain the form of equation (8). The input data for the simulation is summarised in Table 2. Consequently, in the simulations conducted in this paper, the modified Sheppard-Wright law was programmed and used to describe the material behaviour. In the extrusion process, the areas in which small strains occur can be ignored because they do not affect either the pressure or the properties. Equation (8) is therefore adequate to describe the rheology of the material.

During the simulation of extrusion, large deformations are predominant which require a Lagrangian mesh to be defined. The elements become severely distorted during the process and consequently, the need for remeshing is necessary to continue the simulation. The frequency of remeshing is controlled by the degree of deformation and is a user variable. To improve the accuracy of the results, it is critical to control the degree of remeshing in the areas where high deformation is expected. Refinement mesh boxes of Eulerien type (but maintaining Lagrangian flow) were applied to the billet at re-entrant corners to the die where high deformation is expected to occur. A coarser mesh is used for the remainder of the billet. The mesh for the FEM simulations was generated automatically for a space domain that included billet, container and the ram. Simulations were conducted on a Dell dual processor workstation.

\section{METHOD OF IDENTIFYING FRICTION FACTOR}

The extrusion process requires large compressive loads and involves a minimal displacement when first upsetting the ram to fill the container. The simulation output ensures that given the correct data, FEM is able to predict the real material behaviour, which is determined in such a way that conditions are identical to these encountered in industrial practice.

The basic experimental principle of the extrusion process consists of applying a velocity $v$ to a billet heated at an initial temperature $T^{\circ}$ while recording the values of experimental pressure $P^{\mathrm{ex}}$ as a function of the displacement of the ram. The data is obtained from the experimental pressure displacement trace curves obtained for different initial temperatures.

The method consists of changing the friction coefficient $\bar{m}$ in the simulation in order to build the corresponding computed loads $P^{\mathrm{C}}$, for each extrusion temperature. The goal is to determine the friction coefficient that allows the prediction of the experimental data when the computed

Table 1 Chemical compositions of aluminium alloy 2024 and Al- 1 wt-\%Cu (Refs. 16 and 17), wt-\%

\begin{tabular}{llllllll}
\hline Alloy & Al & $\mathrm{Cu}$ & $\mathrm{Si}$ & $\mathrm{Mn}$ & $\mathrm{Mg}$ & $\mathrm{Fe}$ & $\mathrm{Ti}$ \\
\hline AA 2024 & Balance & $3.7-4.5$ & 0.15 & $0.15-0.8$ & $1.2-1.5$ & 0.2 & 0.15 \\
Al-1 wt-\%Cu & Balance & 0.9 & 0.82 & 0.71 & 0.5 & 0.25 & 0.014 \\
\hline
\end{tabular}

Table 2 Rheology data input used for computer simulation ${ }^{16,17}$

\begin{tabular}{llllll}
\hline & \multicolumn{2}{l}{ Flow stress data* } & & \\
\cline { 2 - 5 } Alloy & $\begin{array}{l}\alpha, \mathrm{m}^{2} \\
\mathrm{MN}^{-1}\end{array}$ & $n$ & $\begin{array}{l}\Delta H, \mathrm{~kJ} \\
\mathrm{~mol}^{-1}\end{array}$ & $\ln A$ & $\begin{array}{l}\text { Temperature, } \\
{ }^{\circ} \mathrm{C}\end{array}$ \\
\hline 2024 & 0.016 & 4.25 & 148880 & $19 \cdot 6$ & $300-350-400-450$ \\
$\mathrm{Al}-1 \mathrm{wt} \% \mathrm{Cu}$ & 0.0167 & $5 \cdot 65$ & 147950 & $26 \cdot 7$ & $300-450$ \\
\hline
\end{tabular}

$* \alpha, n$, and $A$ are constants; $\Delta H$ activation energy for deformation. 


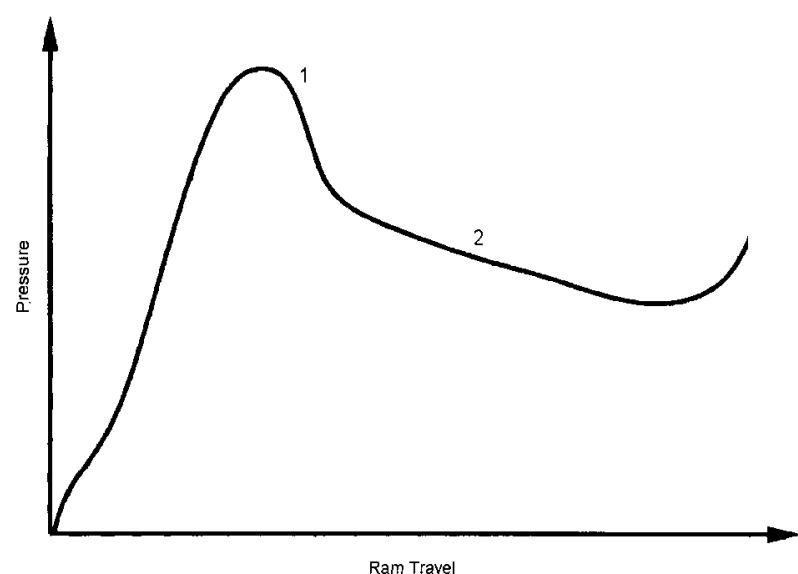

\section{Locations where friction was examined}

results are close or agree with experiment measurements for a specific material and conditions.

Since various factors during the extrusion process require experimental procedures, there are many points at which a direct comparison between the experimental work and its analytical counterpart can be compared and the accuracy of the output assessed. To investigate in greater detail the friction phenomena during the extrusion cycle, two locations of interest were selected for examination and are shown in Fig. 1. The first location identifies the friction at the start of the extrusion after the peak pressure has been established and the second location where the pressure is influenced to a greater extent by the associated temperature rise.

\section{Results and discussion}

\section{EFFECT OF INITIAL BILLET TEMPERATURE ON FRICTION}

Friction conditions at the billet/tool interface are known to affect the flow paths of the material at the container and through the die by changing the extrusion parameters, loads, stresses, and surface quality. Using different friction conditions and various input temperatures permits the experimental pressure to be predicted from the simulation. The predicted loads $P_{\mathrm{m}}^{\mathrm{C}}$ of the corresponding friction coefficients for each extrusion temperature are given in Table 3. By comparing the predicted loads $P_{\mathrm{m}}^{\mathrm{C}}$ with the experimental loads $P^{\mathrm{ex}}$, the results gave a clear indication that the coefficient of friction varies with the initial billet temperature for both aluminium alloys investigated.

The computed results for the variation of the coefficient of friction $\bar{m}$ with temperature are illustrated in Fig. 2. The figure indicates a linear relationship with a good correlation of the form

$$
\bar{m}=A+B . T \text {. }
$$

The figure also illustrates that the difference in friction

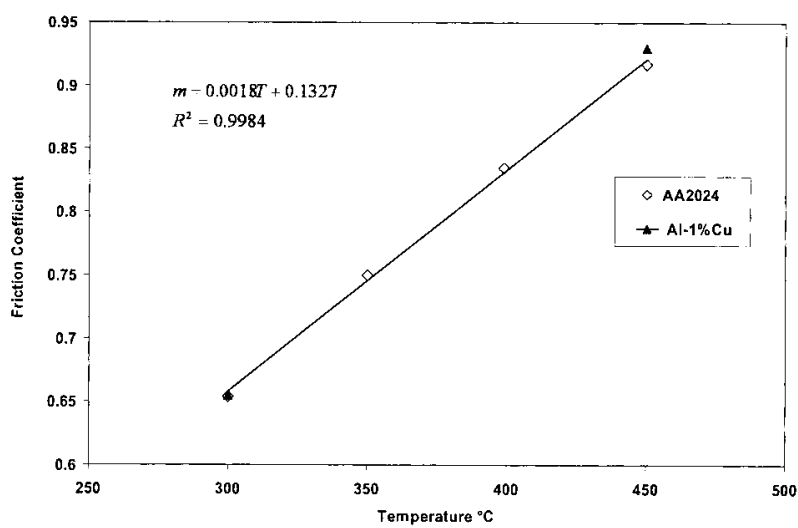

2 Variation of friction coefficient $\bar{m}$ with initial billet temperature

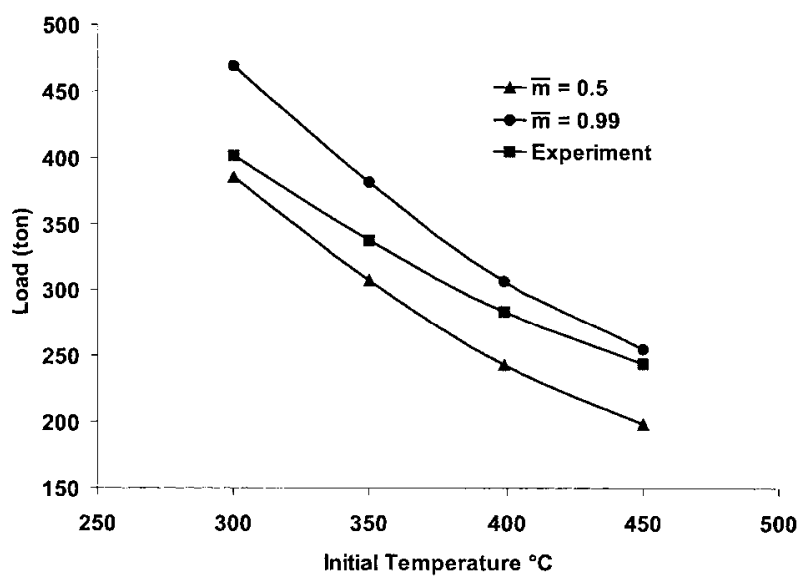

3 Predicted and experimental load variation with initial billet temperature for aluminium alloy 2024: $\bar{m}$ friction coefficient; 1 ton $=1.016 \mathrm{t}$

coefficient between low and high temperature extrusion increases with increasing temperature. The predicted pressures for input temperatures correspond to the extremes of frictional conditions, denoted by $\bar{m}=0.5$ and 0.99 , are shown in Fig. 3. As expected, a general trend of increasing extrusion pressure with decreasing initial billet temperature was found. The experimental points shown in the figure support the thesis that the friction coefficient varies with temperature. Hence, it can be concluded that it will also vary during the ram stroke. The figure also illustrates a greater difference between the experimental and predicted pressure at low and high friction for all extrusion temperatures. The results show that at $300^{\circ} \mathrm{C}$ using sticking friction overestimates the peak pressure significantly. Hence, the assumption that the friction is a constant value for all extrusion temperatures is incorrect. Moreover, the friction coefficient was found to vary from 0.654 for $300^{\circ} \mathrm{C}$ to 0.92 for $450^{\circ} \mathrm{C}$ as given in Table 3 at location (1) (just after the peak pressure has been established).

Table 3 Comparison of predicted loads $P^{C}$ and experimental loads $P^{e x}$ with initial billet temperature for both alloys at location (1)

\begin{tabular}{|c|c|c|c|c|c|c|c|c|c|c|c|c|c|c|c|c|}
\hline \multirow[b]{2}{*}{$\begin{array}{l}\text { Temperature, } \\
{ }^{\circ} \mathrm{C}\end{array}$} & \multicolumn{2}{|l|}{$P_{0.5}^{C}$} & \multicolumn{2}{|l|}{$P_{0.6}^{C}$} & \multicolumn{2}{|l|}{$P_{0.7}^{\mathrm{C}}$} & \multicolumn{2}{|l|}{$P_{0.8}^{\mathrm{C}}$} & \multicolumn{2}{|l|}{$P_{0.9}^{C}$} & \multicolumn{2}{|l|}{$P_{0.99}^{C}$} & \multicolumn{2}{|l|}{$P^{e x}$} & \multicolumn{2}{|l|}{$\bar{m}$} \\
\hline & 2024 & $\begin{array}{l}\mathrm{Al}-1 \\
\mathrm{wt}-\% \mathrm{Cu}\end{array}$ & 2024 & $\begin{array}{l}\mathrm{Al}-1 \\
\mathrm{wt}-\% \mathrm{Cu}\end{array}$ & 2024 & $\begin{array}{l}\mathrm{Al}-1 \\
\text { wt-\%Cu }\end{array}$ & 2024 & $\begin{array}{l}\mathrm{Al}-1 \\
\mathrm{wt}-\% \mathrm{Cu}\end{array}$ & 2024 & $\begin{array}{l}\mathrm{Al}-1 \\
\mathrm{wt}-\% \mathrm{Cu}\end{array}$ & 2024 & $\begin{array}{l}\mathrm{Al}-1 \\
\text { wt-\%Cu }\end{array}$ & 2024 & $\begin{array}{l}\mathrm{Al}-1 \\
\text { wt-\%Cu }\end{array}$ & 2024 & $\begin{array}{l}\mathrm{Al}-1 \\
\text { wt-\%Cu }\end{array}$ \\
\hline 300 & 385 & 456 & 396 & 470 & 414 & 488 & 432 & 510 & 448 & 536 & 469 & 569 & 401 & 458 & 0.654 & 0.655 \\
\hline 350 & NA & NA & NA & NA & 332 & NA & 346 & NA & 362 & NA & 381 & NA & 338 & NA & 0.75 & NA \\
\hline 400 & NA & NA & NA & NA & NA & NA & 268 & NA & 279 & NA & 306 & NA & 284 & NA & 0.835 & NA \\
\hline 450 & NA & NA & NA & NA & NA & NA & NA & NA & 242 & 275 & 256 & 284 & 244 & 280 & 0.917 & 0.93 \\
\hline
\end{tabular}



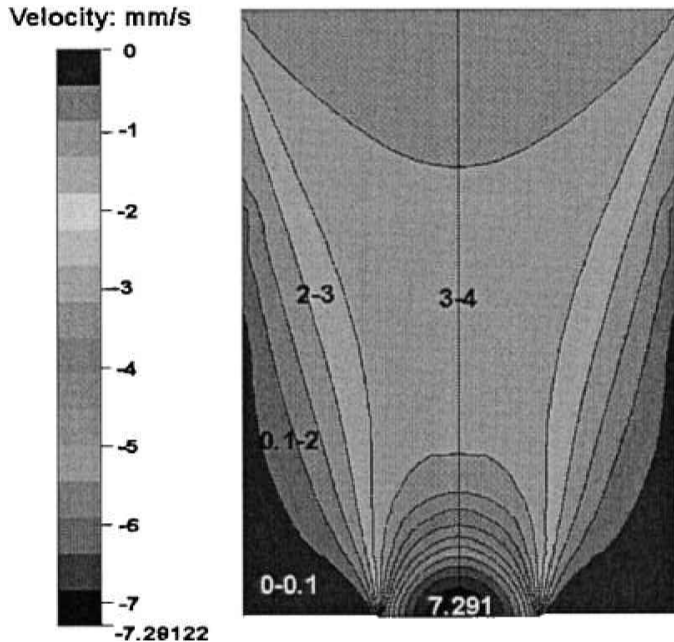

a

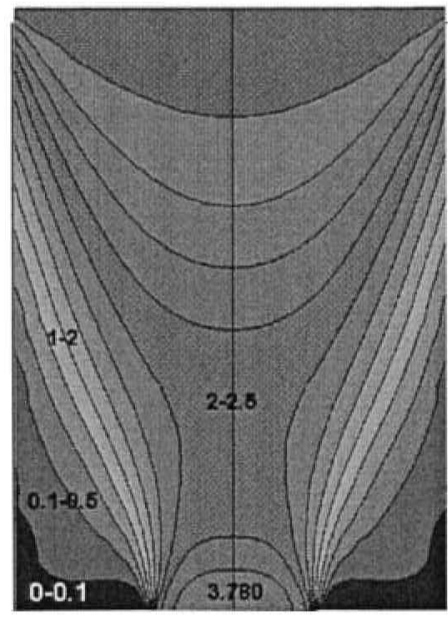

b

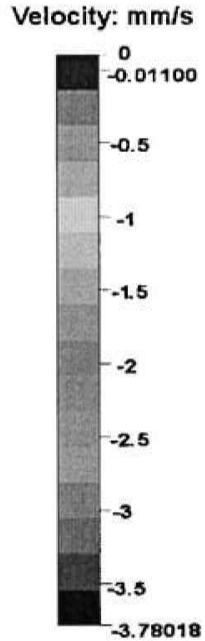

3.78018

a $450^{\circ} \mathrm{C} ;$ b $300^{\circ} \mathrm{C}$

\section{Initial contact area at 450 and $300^{\circ} \mathrm{C}$ extrusion}

Before extrusion can proceed, the billet must be inserted into the container and to facilitate entry there is generally appreciable clearance between the two. At this initial setup, the only contact of the billet with the tools is at the die face where the boundary conditions including friction have no direct effect on the deformation. The necessary force needed to be applied to cause the billet to fill the clearance is affected predominantly by the nature of the material and the manner in which its properties are affected by the temperature, i.e. material flow stress and the rate at which the work is carried out. The first effect of pressure at this stage is thus to compress or upset the billet into firm contact with the cylinder wall. The upsetting stages, before location (1) has been reached for the billet at high and low temperature extrusions are shown in Fig. 4 represented by the prevailing velocity profiles. The pressure at this stage rises sharply while the billet is being compressed to fill the container. As the figures illustrate, at higher temperature extrusion, the deformation is characterised by more rapid flow. The velocity at which the material flows to fill the space of the container and a move towards the die opening is significantly greater compared with that at lower temperature. This is evident since the resistance to deformation of the billet material decreases as the temperature increases and reaches its lowest permissible value when the material is close to the melting point of the alloy's lowest melting phase (i.e. $511^{\circ} \mathrm{C}$ for 2024 ). However, there are significant differences in the rate as a result of which this change occurs and in the ultimate degree of ductility, which is reached. Consequently at higher temperature extrusion, the billet is more ductile and tends, under deformation, to spread more easily to fill the container. The deformation of the billet at this initial stage is characterised by nearly half of its length sticking along the container wall as illustrated in Fig. $4 a$. On the other hand, at lower temperature extrusion, the billet is less ductile and acts in a relatively stiffer manner. The upsetting stage at this temperature extrusion is initiated by sliding along most of the container wall and only one fifth of the billet length is sticking as shown in Fig. $4 b$. The shapes of the dead metal zones (DMZs) are also quite different at these varying temperatures. This initial contact and the extent of this contact area along the container wall have a significant effect on the commencement of the deformation path which occurs in localised regions in the billet where the interface results in a stick, slip or fully sticking friction situations before the quasi-static DMZ formation. Moreover, when the billet has been fully compressed to fill the container, the elastic properties of the container and plastic properties of the materials in contact, have a significant effect on the difficulty of the two materials to slide relative to each other. Similarly, the plastic work dissipated depends on the local temperature at the contact area and the thermal conductivity for the two materials in contact, i.e. billet/container. As a result of the extent of the area of contact, at higher temperature and lower flow stress, the force to deform the material increases as the contact pressure at the container increases. In addition, at lower temperature extrusion (i.e. $300^{\circ} \mathrm{C}$ ), the surfaces in contact would produce a greater elastic deformation in the container owing to the higher flow stress.

After further ram travel, the billet is further compressed and a very small amount of extrusion takes place. The DMZ is fully established by this time. However, the deformation zone is not clearly defined at this stage until the peak pressure reaches its maximum. The deformation zone is not stationary, the billet is deforming plastically while the shearing forces are operating which promotes the burnishing effect at the DMZ/billet interface. It is at this stage, after the peak pressure has been established, that the friction is of great interest. Not only because of press capacity requirement, but also because of its contribution to the temperature changes occurring in the material whilst reaching the peak pressure.

In order to relate the friction factor through a relationship applicable for all extrusion variables and to overcome the separate relationships generated for the variation of friction coefficient with temperatures as stated in equation (1), it would therefore be more instructive to incorporate the friction factor into a sinh relationship by using the $\ln Z$ parameter. The parameter $Z$ defined in equation (8) contains the data describing the alloy behaviour (Table 2), ram speed and temperature. The temperature compensated strain rate just after the peak pressure is established, $Z_{\mathrm{d}}$ at location (1), incorporating both strain rate and temperature should enable the friction variation to be related by one relationship. The strain rate varies from point to point throughout the deformation zone during the extrusion process. It is thus necessary to use an average value for this parameter. The mean equivalent strain rate used in this investigation is calculated from an average area over the deformation zone where the maximum deformation is expected to occur. The parameter $Z_{\mathrm{d}}$ was programmed and incorporated into FORGE2 and FORGE3 by the authors as one of the outputs of the simulation. The parameter $Z_{\mathrm{d}}$ 


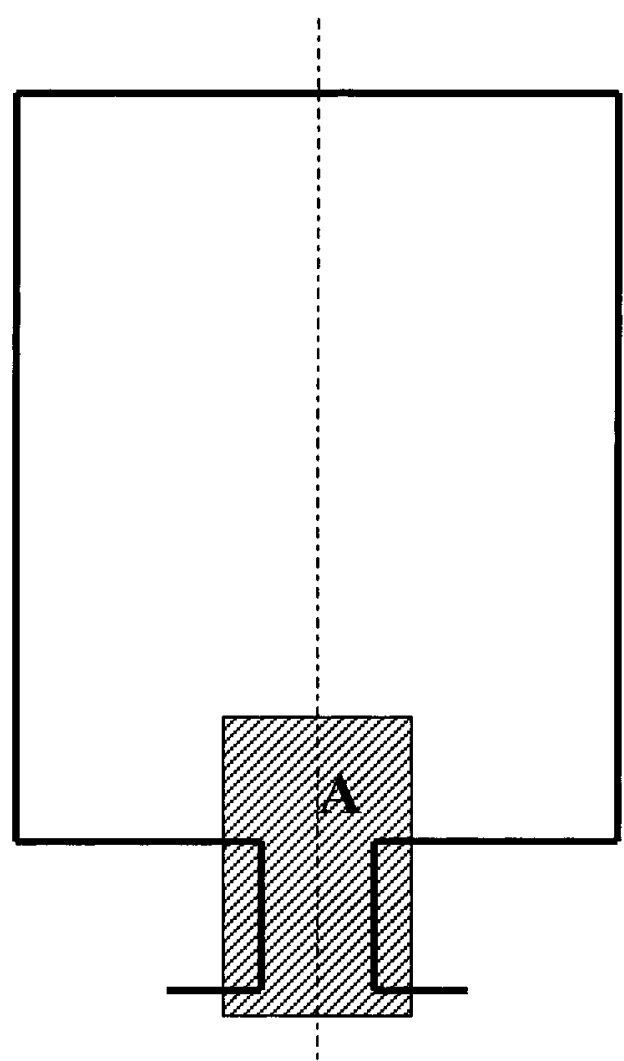

5 Boundary area where values of $Z_{d}$ were averaged: $Z_{d}$ temperature compensated strain rate just after peak pressure is established

used in this investigation is calculated in the same manner as the mean equivalent strain rate at an instantaneous maximum temperature as illustrated in Fig. 5. To ensure different alloys to be represented by the same mathematical expression and to account for the effect of chemical composition of the materials, $\ln \left(Z_{\mathrm{d}} / A\right)$ together with the function $\alpha n$ was used ${ }^{1}$ as proposed by Sheppard for pressure calculations. The constant $\alpha$ is a reciprocal flow stress, and its value has been interpreted as the position where the dependence of flow stress upon strain rate changes from, a power relationship to an exponential one, and is a function of material chemistry. The constant $n$ can be termed the inverse of the temperature compensated strain rate sensitivity. The actual value of this constant is strongly dependent upon the value of $\alpha$. Both are intimately related to the chemical composition of the material.

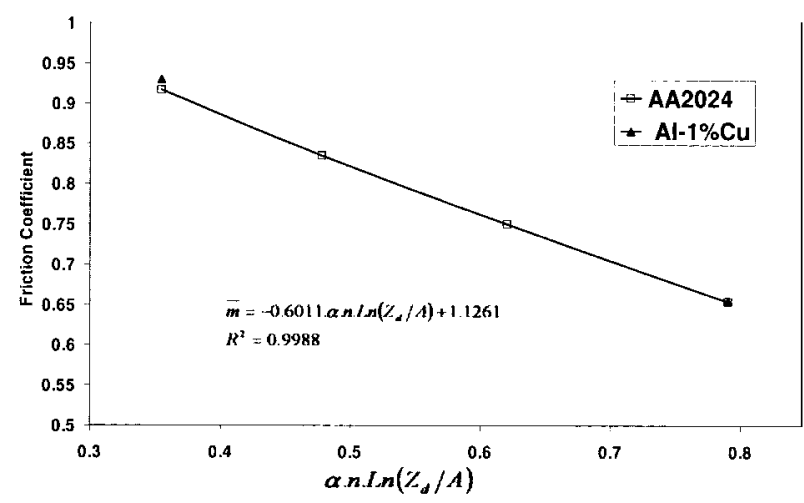

6 Friction variation with $\alpha n \ln \left(Z_{d} / A\right)$ at location (1): $\bar{m}$ friction coefficient; $Z_{\mathrm{d}}$ temperature compensated strain rate just after peak pressure is established; $A, \alpha$, and $n$ are constants
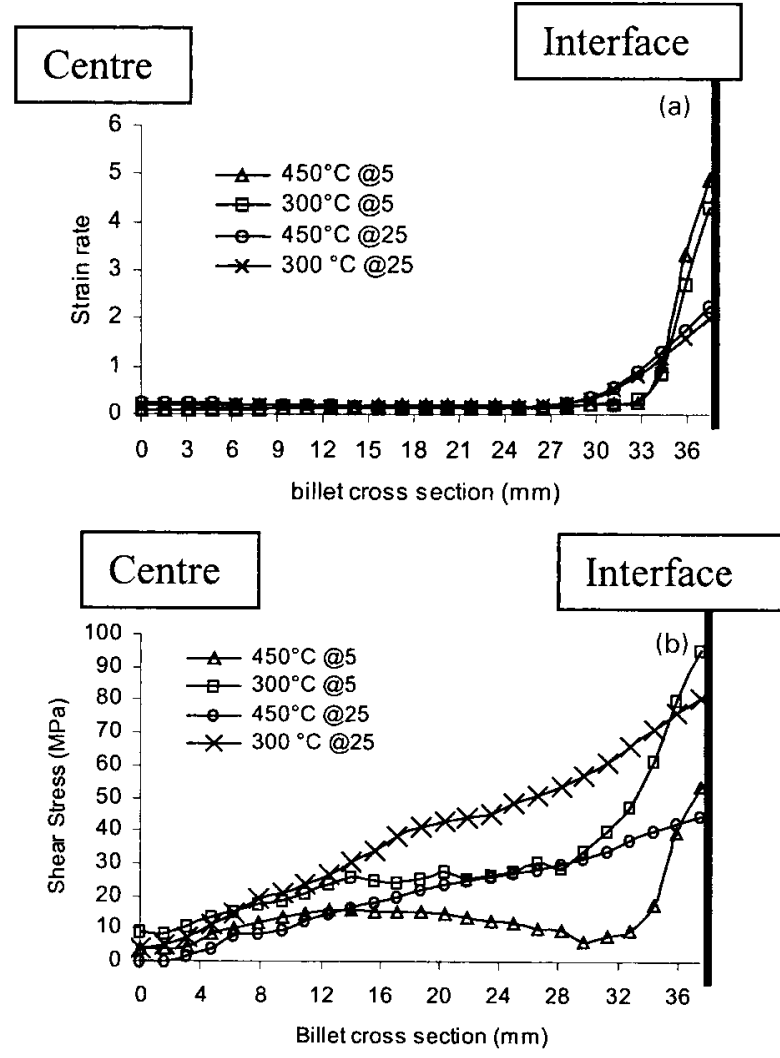

a strain rate, 5 and $25 \mathrm{~mm}$ are distances from ram position after peak pressure had been achieved; $b$ shear stress, at $5 \mathrm{~mm}$ from ram

7 Strain rate and shear stress variations across billet cross-section

When friction coefficients are plotted against $\alpha n \ln \left(Z_{\mathrm{d}} / A\right)$ a linear relationship of the form

$\bar{m}=-0 \cdot 6011 \alpha n \ln \left(Z_{\mathrm{d}} / A\right)+1 \cdot 1261$

is apparent and is shown in Fig. 6. The resistance of the material to withstand the imposed strain gradients at low $Z_{\mathrm{d}}$ conditions, i.e. low flow stress, results in a larger deformation zone extending to the rear of the billet whilst at high $Z_{\mathrm{d}}$ conditions, the high flow stress restricts the deformation zone to the die mouth region. As shown in Fig. $7 a$ and $b$, at $5 \mathrm{~mm}$ from the ram face, high deformation occurs such that there is a shear zone at the billet/container interface in which the strain rate and the shear stress vary from a higher value to zero for the strain rate and to a minimum value for the shear stress at the centre of the billet. At $25 \mathrm{~mm}$ distance from the ram, the decay of both the shear stress and the strain rate when moving from interface to billet centre is much more gradual. This is clearly owing to the absence of deformation at the rear of the billet in the first case and the interaction with the DMZ and the position of greater deformation as the material approaches the die. When approaching the die, the zone of intense shear increases toward the die opening, separating the deformation zone from the DMZ. In this case, the frictional stress is identical to the flow stress of the billet material in pure shear, whereas the relative speed increases from the ram speed at the rear end of the billet to approach the exit speed of the extrudate when approaching the die opening.

Another factor that must be considered is the strain rate. Although the total strain is the same for each case, i.e. in terms of the reduction, the time in which the heavy deformation zones form is different. Since the deformation is initiated nearer the rear of the billet in the high temperature case, over a longer contact area, there will be an 


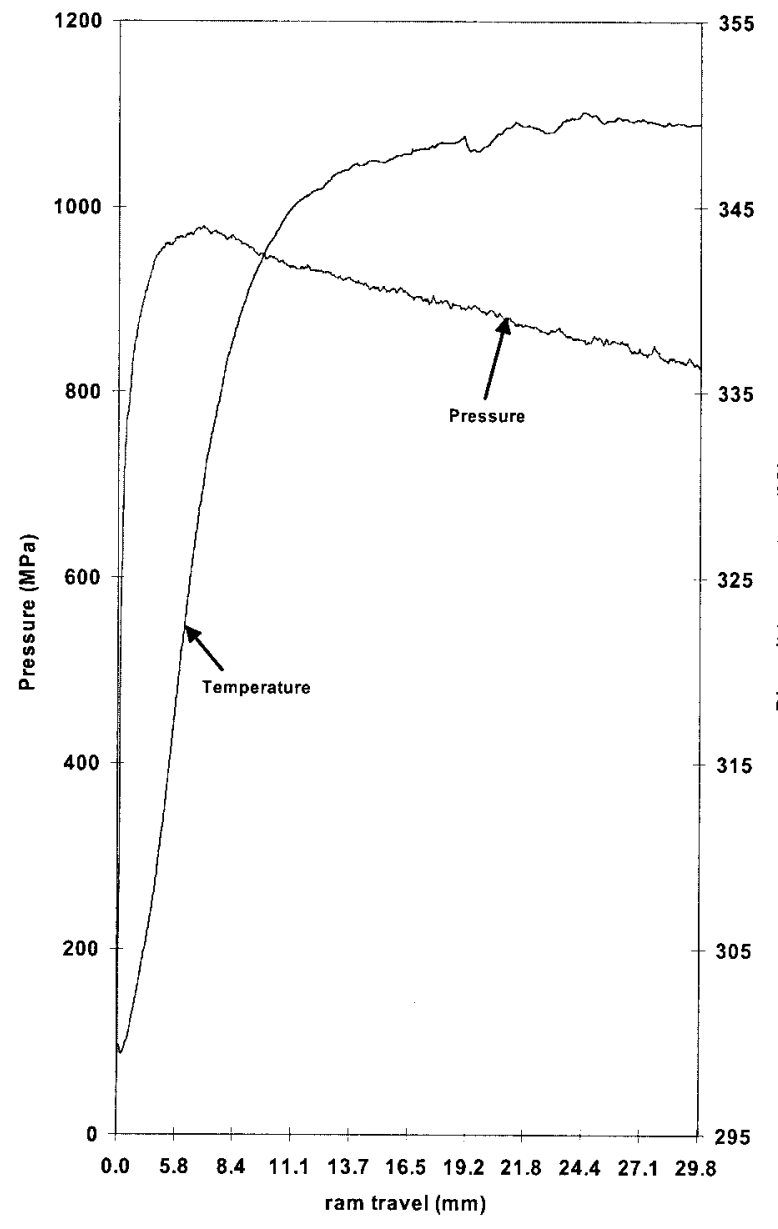

8 Variation of billet temperature with pressure

associated greater time available for the major deformation to be completed resulting in a lower strain rate. Thus the friction coefficient is raised and approaches a unit value. In terms of the material plastic flow behaviour, the observations are not unrealistic.

However these values of friction do not remain constant through the extrusion process. These changes are the consequences of an increase in the overall billet temperature within the deformation zone and the decrease of the area of contact with further ram travel. Complex thermal changes begin as soon as the extrusion commences. The most critical temperature is clearly the die exit temperature of the extrudate, which is related to the heat balance history commencing at the upsetting stage of the operation. It increases if the heat produced by deformation and friction exceeds the heat losses and decreases if the reverse is true. The exit temperature increases as the peak pressure is reached and appears to remain approximately constant after $\sim 30 \mathrm{~mm}$ ram travel (see Fig. 8). The figure illustrates the variation of pressure displacement with the exit die temperature. For the initial temperature of $300^{\circ} \mathrm{C}$, the temperature of the billet increased sharply by $27 \mathrm{~K}$ in reaching the peak pressure. This temperature rise would be expected as a result of the work done in upsetting the billet, and the work done against friction between the material and the tooling. This is caused by the work done as the material is forced to commence flow through the die orifice owing to the production of heat by deformation being transferred to material at the inlet of the die from the deformation zone. This heat is generated in the deformation zone and influenced by the friction coefficient. Clearly this would produce an increase in the extrusion temperature. Since the work done in overcoming friction is a function of the shear

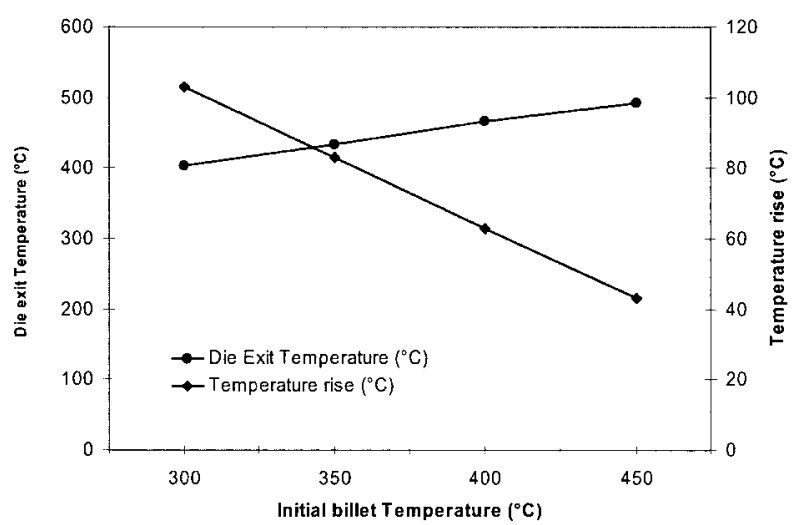

9 Predicted die exit temperature rise

stress within the deformation zone, then the pressure needed to overcome the friction must also be a function of the temperature differential $\Delta T$ between the initial and actual billet temperature, which produces a decrease in flow stress. However, the value of $\Delta T$ depends on the initial billet temperature of the material as shown in Fig. 9. The exit temperature increases with increasing initial billet temperature and the temperature rise $\Delta T$ decreases as the billet temperature increases, resulting in a decrease in the material flow stress. Taking into account the temperature rise $\Delta T$ within the deformation zone at location (2), it was found that the friction coefficient increases from 0.65 to 0.89 for the initial billet temperature of $300^{\circ} \mathrm{C}$ and from 0.91 to 0.98 for $450^{\circ} \mathrm{C}$ as shown in Fig. 10. Similar to location (1), an

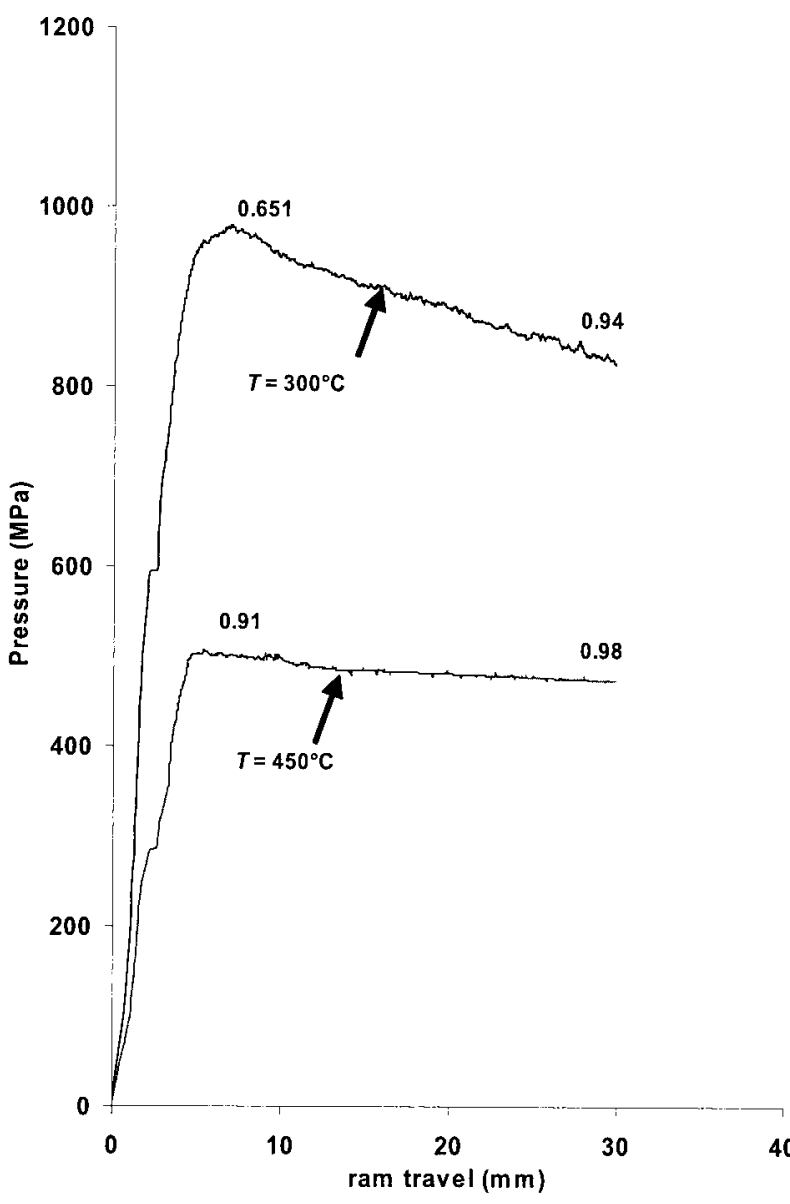

10 Friction variation during extrusion process for aluminium alloy 2024 at locations (1) and (2); temperatures given are initial billet temperatures 


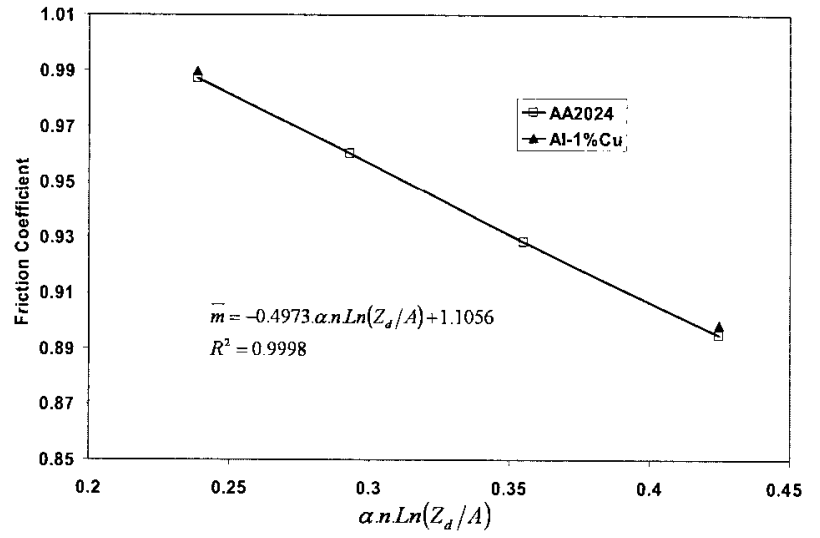

11 Friction variation with $\alpha n \ln \left(Z_{d} / A\right)$ at location (2): $\bar{m}$ friction coefficient; $Z_{\mathrm{d}}$ temperature compensated strain rate just after peak pressure is established; $A$ $\alpha$, and $n$ are constants

apparent linear relationship of the form

$$
\bar{m}=-0 \cdot 4973 \alpha n \ln \left(Z_{\mathrm{d}} / A\right)+1 \cdot 1056
$$

was found when the coefficient of frictions were plotted against $\alpha n \ln \left(Z_{\mathrm{d}} / A\right)$ and is shown in Fig. 11. Figures 6 and 11 indicate that over the range of $\ln \left(Z_{\mathrm{d}} / A\right)$ considered, the function relating $\bar{m}$ and $\alpha n \ln \left(Z_{\mathrm{d}} / A\right)$ are of similar form. The high correlation obtained throughout the analysis indicates that the relationship is applicable to all temperatures. The results show that the use of the $Z_{\mathrm{d}}$ parameter is justified, since for a given value of $Z_{\mathrm{d}}$, the pressure necessary to carry out extrusion increases with reduction ratio as predicted by previous workers. ${ }^{1,16}$ At the initial stages of the process, the main parameters affecting the friction are the increase in the temperature and the contact length. However, near the end of the process, the contact length has diminished as the length of the billet shortens. It was not feasible to predict the coefficient of friction after location (2) because of computer time considerations. However, there is no indication that such an operation would alter the interpretation of the data already collected. At all locations, the total friction force is significantly influenced by the billet length remaining in the container, and thus falls as extrusion proceeds.

Hence, the only method of predicting friction could be to program the FEM code to define friction as

$$
\bar{m}_{\Delta \mathrm{L}_{\mathrm{n}}}=\left[A+\alpha n \ln \left(Z_{\mathrm{d}} / A\right)+B\right]_{\Delta \mathrm{L}_{\mathrm{n}-1}}
$$

where $\Delta L_{\mathrm{n}}$ is incremental ram travel (i.e. each $2.5 \mathrm{~mm}$ of ram displacement).

The authors realise that programming a boundary condition in this way would not be a trivial task and would increase computer time considerably. Nevertheless, if friction is to be addressed in a scientific manner such an approach will be necessary.

\section{EFFECT OF INITIAL BILLET TEMPERATURE ON MATERIAL FLOW}

To evaluate the effects of material flow at both high and low temperature extrusion i.e. 450 and $300^{\circ} \mathrm{C}$ respectively, two billets were sectioned along a meridian plane with square grid lines. Similar grid lines were simulated in order to compare with the experimental results. ${ }^{23}$

The effects of the initial billet temperature on material flow for high and low temperature extrusion are illustrated in Fig. 12. The figure shows a comparison between the simulated gridded billets at the end of the extrusion (Fig. $12 a$ and $b$ ) and the macrographs taken from experiments (Fig. 12c and $d$ ). ${ }^{23}$ A good agreement between the experiments and the simulation is obvious. As these figures illustrate, it is clearly evident in both cases that there is a significant change in deformation zone geometry. For high temperature extrusion (i.e. $450^{\circ} \mathrm{C}$ ), the deformation zone gradually spreads from above the die mouth to the
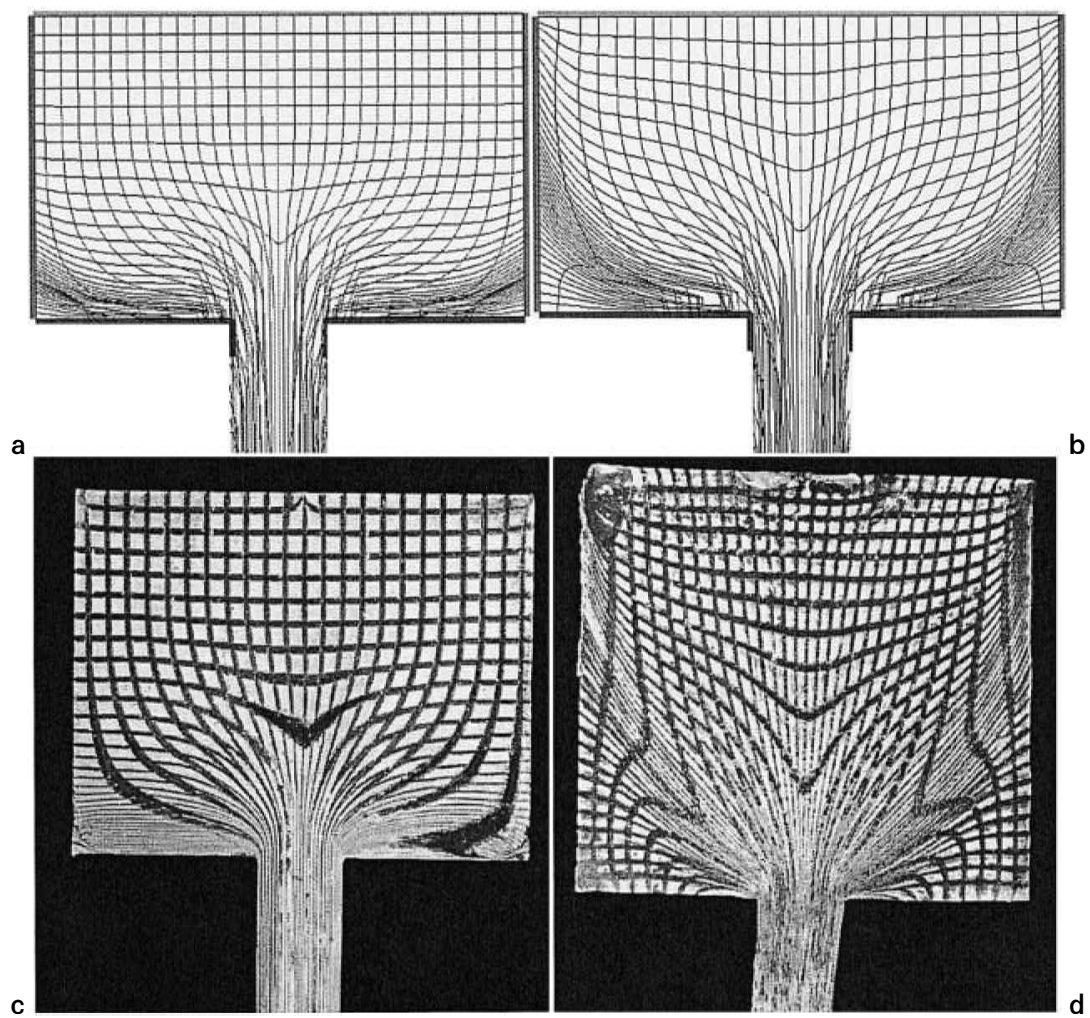

a flow at $300^{\circ} \mathrm{C} ; b$ flow at $450^{\circ} \mathrm{C} ; c$ and $d$ are corresponding experimental ${ }^{23}$ flow patterns at 300 and $450^{\circ} \mathrm{C}$ respectively 12 Simulated flow patterns at end of extrusion 


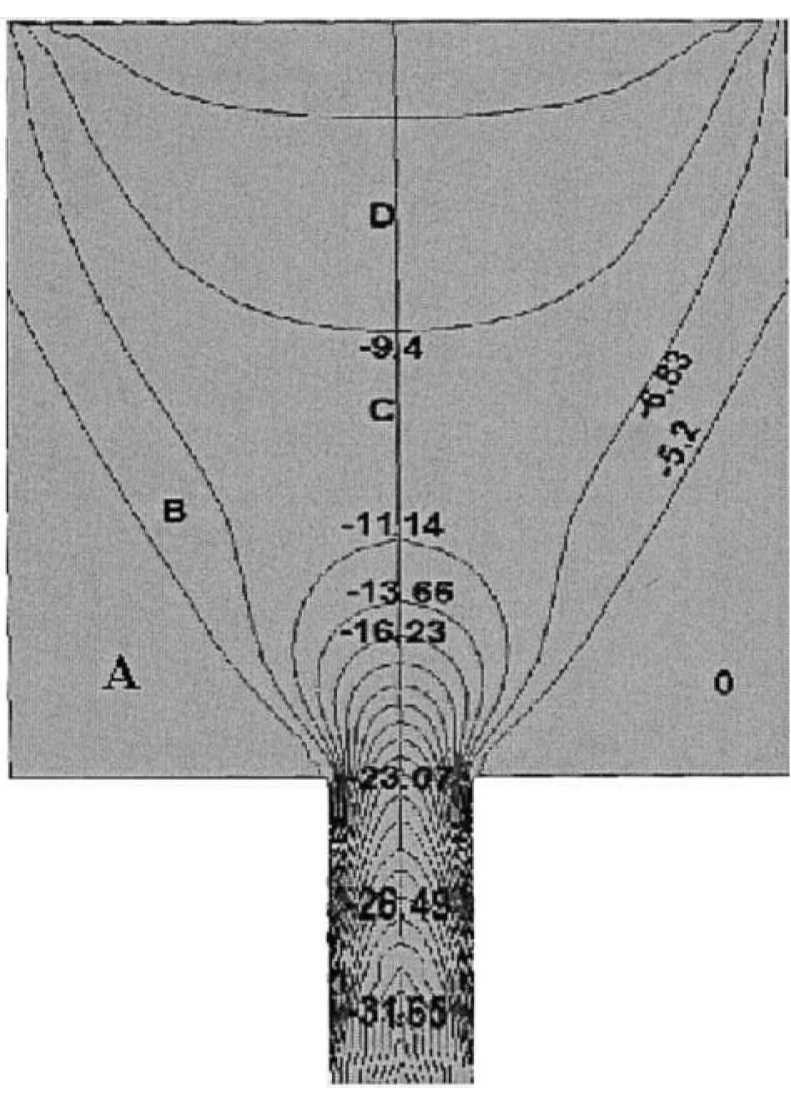

(A) dead metal zone; (B) surface generation zone; (C) main deformation zone; (D) central deformation zone

\section{Flow velocity of material during extrusion at $450^{\circ} \mathrm{C}$}

container walls by progressive thickening of the zones of heavy shear. The shearing zone occurs in the subcutaneous regions of the billet in preference to sliding at the container wall interface. On the other hand for lower temperature conditions (i.e. $300^{\circ} \mathrm{C}$ ), the extent of this zone is volumetrically less than that observed with higher temperature conditions, the most severe deformation is limited to a small volume close to the die mouth. The formation of the DMZs is evident and over at least $50 \%$ of the volume shown there is no evidence of deformation, the grid is regular across the entire width. Comparing this with the result of the high temperature extrusion shows a considerable difference. In this case, the effects of the deformation can be found over the entire surface shown. Considerable amounts of cylinder wall shear can be seen stretching from the rear of the billet up to the DMZ boundary and progressing further back into the billet, leaving the die mouth flow at a more obtuse angle to the DMZ than the one observed at lower temperatures. The zone of shear produced by friction at a temperature of $450^{\circ} \mathrm{C}$ is greater in depth than the one formed at $300^{\circ} \mathrm{C}$. The shearing at the DMZ boundary can be regarded as a measure of the work required to provide the force needed to produce a satisfactory surface. This work increases with an enlarged DMZ as a result of the increase of the initial billet temperature and consequent increase in the friction factor at the billet/container interface.

Therefore, the extent of the DMZ provides some understanding of the influence of temperature on the friction and can be visualised by assessing the velocity profiles. As illustrated by Fig. 13, in the regions of the deformation zone close to the die, the central velocity is greater. The boundaries of the deformation zones illustrated in Fig. 12 shows a smaller volume for the lower temperature extrusion. In addition there is a greater amount of material from the billet subcutaneous layers appearing to reach the extrudate in the high temperature case than the low temperature. This would tend to imply greater diffusion of internal energy dissipation. The temperature gradient between the centre and the container wall will also cause the shearing layer between the billet and the container to occur at a greater depth below the surface, and so leave a thicker rim of metal at the billet/container interface. In this connection, the strong adherence of aluminium at lower flow stress to the wall of the container would eventually lead to a very high value of $\bar{m}$.

Thus, the evidence confirms that a lower temperature extrusion increases the resistance to deformation, reduces the volume of DMZ and decreases the proportion of the work required to produce the desired surface. Therefore, the friction factor is associated with the thermally activated events involved. The results found in Figs. 6 and 11 of a decreasing $\bar{m}$ with increasing $\alpha n \ln \left(Z_{\mathrm{d}} / A\right)$ term can therefore be attributed to a change in deformation zone geometry caused by a variation in the plastic flow behaviour of the material.

In order to achieve a sound quality surface during the extrusion process, the surface of the extrudate must be generated from within the billet. The generation of this surface involves the production of a virgin surface by shearing of the DMZ. The nature of shear involves production of continuous virgin surfaces at the entrance to the die throat. However, the boundary conditions at the billet/ container interface, i.e. friction, heat transfer etc., have a significant effect on the material which constructs the surface of the extrudate.

The quasi-static flow of material during extrusion is shown in Fig. 13. The conditions at the billet interface wall have promoted the formation of a DMZ at the front of the billet. This static volume of material (A) extends from the die throat, back towards the container wall at an angle, which depends on the initial billet temperature and the boundary conditions at the interface. Outlining this zone is a region of intense shear. The zone resembles the frustum of a cone in shape and extends from the die throat to the container wall. It can be seen from the figure that the extrudate material originates from various locations in the deforming billet that can be represented by three zones. The surface generation zone (B) outlining the DMZ, the main deformations zone $(C)$ and the central deformation zone (D). The outer surface of the extrudate originates from the surface generation zone (B) as illustrated by Fig. 13. It is material from this zone moving along the DMZ boundary that forms the extrudate surface. The figure also suggests that the outside surface of the extrudate originates from the material which is located on the edge of the DMZ and moving more slowly into the extrudate surface promoting a continuous formation of a virgin surface. The material forming the core of the extrudate originates from the central deformation zone (D) of heavy shear feeding into the bulk of the extrudate. This central deformation zone supplements the material feeding the body of the extrudate from within the main deformation zone (C).

\section{Conclusions}

1. The initial stages of the deformation, where the billet is primarily affected by the flow stress of the material and the rate, at which the work is carried out, proved to be of some significance in dictating the material flow distribution during the extrusion process.

2. The assumption that the friction conditions are a constant value for all extrusion temperatures is incorrect. The apparent friction coefficient was found to vary from 0.654 for $300^{\circ} \mathrm{C}$ to 0.92 for $450^{\circ} \mathrm{C}$.

3 . The $\bar{m}$ values do not remain constant throughout the extrusion cycle. The increases in overall temperature of the billet and hence decreases in flow stress, lead to an increase in friction for both alloys and temperatures. 
4. The variation of friction conditions during the extrusion cycle for both alloys relating the process conditions, resulted by relationships of the form $\bar{m}=-0.6011 \alpha n \ln \left(Z_{\mathrm{d}} /\right.$ $A)+1 \cdot 1261$ at location (1) and $\bar{m}=-0.4973 \alpha n \ln \left(Z_{\mathrm{d}} / A\right)+$ $1 \cdot 1056$ at location (2). This implies that friction should be introduced into the computer program in the form of

$$
\bar{m}_{\Delta \mathrm{L}_{\mathrm{n}}}=\left[A+\alpha n \ln \left(Z_{\mathrm{d}} / A\right)+B\right]_{\Delta \mathrm{L}_{\mathrm{n}-1}}
$$

5. For an accurate simulation of extrusion, the friction coefficient must be identified continuously during the process cycle. This is not only required for press capacity, but also the effect on the temperature changes occurring in the material. This subsequently has an effect on the surface quality of the extrudate.

6. During the extrusion process, the billet surface adheres to the container and affects the possibility of surface layer incorporation into the extrudate. Using a constant value for friction for all extrusion temperatures, as many workers have indicated, would affect the incorporation of the surface layers into the extrudate. This would directly affect the predicted minimum discard.

7. The accuracy of the friction factor $\bar{m}$ is largely dependent upon the flow stress of the material at the billet/ container interface.

8. A good agreement between the experimental and simulated results was found for high and low temperature extrusion flow. An enlarged dead metal zone was observed as a result of the increase in the initial billet temperature and in turn yielded an increase in friction factor.

\section{Acknowledgements}

The authors wish to thank and recognise excellent contributions to the experimental work by Dr M. G. Tutcher, Dr R. P. Vierod, and Dr J. Subramaniyan.

\section{References}

1. T. SHEPPARD: 'Extrusion of aluminium alloys'; 1999, Dordrecht, Kluwer Academic Press.
2. I. FLITTA and T. SHEPPARD: Proc. 7th Int. Seminar on 'Aluminium extrusion technology', Chicago, 197-203; 2000, Washington, DC, The Aluminium Association.

3. I. FLITTA and T. SHEPPARD: Proc. 5th Int. ESAFORM Conf., Krakow, Poland, April 2002, European Scientific Association for Material Forming, 435-438.

4. T. Chanda, J. ZHOU, L. KOWALSI and J. DUSzCZYK: Sci. Mater., 1999, 41, 195-202.

5. B. J. E. VAN RENS, W. A. M. BRELEMANS and F. P. T. BAAJENS: Proc. 7th Int. Seminar on 'Aluminium extrusion technology', Chicago, 99-107; 2000, Washington, DC, The Aluminium Association.

6. T. A. DEAN and Z. M. HU: Proc. 6th Int. Conf. on 'Technology of plasticity', Nuremburg, Germany, September 1999, Vol. 1, 541-550; Springer - Verlag.

7. S. ABTAhi, T. Welo and S. STORen: Proc. 6th Int. Seminar on 'Aluminium extrusion technology', Chicago, 125-131; 1996, Washington, DC, The Aluminium Association.

8. T. Welo, T. S. ABTAHI and I. SKAUVIK: Proc. 6th Int. Seminar on 'Aluminium extrusion technology', Chicago, 101-106; 1996 , Washington, DC, The Aluminium Association.

9. L. ANAND: Comput. Mech., 1993, 12, 197-213.

10. L. ANAND and w. TONG: Ann. CIRP, 1993, 42, 361-366.

11. M. P. CLODE and T. SHEPPARD: Mater. Sci. Technol., 1990, 6, $755-763$.

12. T. CHANDA, J. ZHOU, L. KOWALSI and J. DUSZCZYK: Proc. 7 th Int. Seminar on 'Aluminium extrusion technology', Chicago, 125-134; 2000, Washington, DC, The Aluminium Association.

13. R. J. DASHWOOD and H. B. MCSHANE: Proc. 6th Int. Seminar on 'Aluminium extrusion technology', Chicago, 331-339; 1996, Washington, DC, The Aluminium Association.

14. A. HeEge, P. Alart and E. ONATE: Eng. Comput., 1995, 12, $41-$ 656.

15. D. Y. YANG: Ann. CIRP, 1994, 43, 229-233.

16. J. SUBRAMANIYAN: $\mathrm{PhD}$ Thesis, Imperial College, London, 1989.

17. R. P. VIEROD: PhD Thesis, Imperial College, London, 1983.

18. T. SHEPPARD and S. J. J. PATTERSON: Mech. Work. Technol., $1982,4,39-56$

19. J.-L. CHENOT et al:: Int. Conf. on 'Forging and related technology’ (ICFT 98), 113-122; 1998, Suffolk, Professional Engineering.

20. 'Software manual,' FORGE3 Version 5.3 Transvalor SA, Sophia Antipolis, France, 2001.

21. C. M. SEllars and D. TEGART: Int. Met. Rev., 1972, 1, 17.

22. T. SHEPPARD and D. WRIGHT: Met. Technol., 1979, 13, 215-223.

23. M. G. TUTCHER: PhD Thesis, Imperial College, London, 1979. 